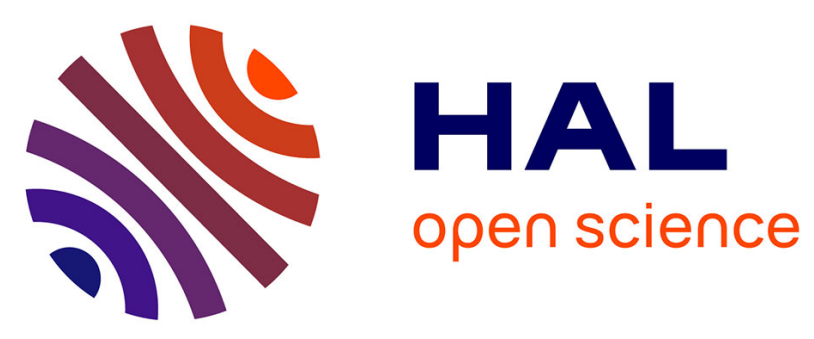

\title{
Quantum phase transitions
}

\author{
M. Lavagna
}

\section{To cite this version:}

M. Lavagna. Quantum phase transitions. Philosophical Magazine B, 2009, 81 (10), pp.1469 - 1483. 10.1080/13642810108208565 . hal-01896128

\section{HAL Id: hal-01896128 https://hal.science/hal-01896128}

Submitted on 16 Oct 2018

HAL is a multi-disciplinary open access archive for the deposit and dissemination of scientific research documents, whether they are published or not. The documents may come from teaching and research institutions in France or abroad, or from public or private research centers.
L'archive ouverte pluridisciplinaire HAL, est destinée au dépôt et à la diffusion de documents scientifiques de niveau recherche, publiés ou non, émanant des établissements d'enseignement et de recherche français ou étrangers, des laboratoires publics ou privés. 


\section{Philosophical}

Magazine

Fint Publithed in n>ys

Philosophical Magazine B

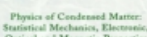

ISSN: 1364-2812 (Print) 1463-6417 (Online) Journal homepage: http://www.tandfonline.com/loi/tphb20

\section{Quantum phase transitions}

\section{Lavagna}

To cite this article: M. Lavagna (2001) Quantum phase transitions, Philosophical Magazine B, 81:10, 1469-1483, DOI: 10.1080/13642810108208565

To link to this article: https://doi.org/10.1080/13642810108208565

曲 Published online: 25 Aug 2009.

Submit your article to this journal

山 Article views: 80

4 Citing articles: 2 View citing articles 진 


\title{
Quantum phase transitions
}

\author{
M. LAVAGNA $\dagger$ \\ Commissariat à l'Energie Atomique, Départment de Recherche Fondamentale \\ sur la Matière Condensée/SPSMS $\neq, 17$ rue des Martyrs, 38054 Grenoble Cedex 9 , \\ France
}

[Received 3 January 2001 and accepted 26 January 2001]

\begin{abstract}
We give a general introduction to quantum phase transitions in strongly correlated electron systems. These transitions, which occur at zero temperature when a non-thermal parameter $g$ such as the pressure, chemical concentration or magnetic field is tuned to a critical value, are characterized by a dynamic exponent $z$ related to the energy and length scales $\Delta$ and $\xi$. We show how one can derive an effective bosonic model associated with fluctuations in the ordering fields. Simple arguments based on an expansion to first order in the effective interaction $u$ allow us to define an upper critical dimension $D_{\mathrm{c}}=4$ (where $D=d+z$ and $d$ is the spatial dimension) below which the mean-field description is no longer valid. We present an alternative tricritical crossover approach valid at $D<D_{c}$ in the large- $N$ limit. We emphasize the role of perturbative renormalization group approaches and self-consistent renormalized spin fluctuation theories in understanding the quantum-classical crossover in the vicinity of the quantum critical point with generalization to the Kondo effect in heavy-fermion systems. Finally, we quote some recent inelastic neutron scattering experiments performed on heavy fermions which lead to an unusual scaling law in $\omega / T$ for the dynamical spin susceptibility, revealing critical local modes beyond the itinerant magnetism picture. We mention new attempts to describe this local quantum critical point.
\end{abstract}

\section{$\S 1$. INTRODUCTION}

Recent years have seen a renewal of interest in the study of quantum phase 1ransitions motivated by experiments in cuprate superconductors, heavy-fermion compounds, organic conductors and related systems (Coleman et al. 1996, Sachdev 1999). By definition a quantum phase transition is a phase transition which occurs at zero temperature when a non-thermal parameter $g$ such as the pressure, chemical concentration or magnetic field is tuned to a critical value. The transition is characterized by quantum fluctuations even in the ground state owing to the Heisenberg uncertainty principle. It is usually accompanied by a drastic change in the behaviour of the correlation functions.

Here, we shall focus on the quantum phase transitions of second order. The associated fluctuations are characterized by two different quantities that are respectively the energy scale $\Delta$ and the correlation length $\xi$. In the case when the energy spectrum has a gap, $\Delta$ measures the difference between the energies of the ground

$\dagger$ Email: lavagna@cea.fr

$\ddagger$ Also part of the Centre National de la Recherche Scientifique.

Philosophical Magazine B ISSN 1364-2812 print/ISSN 1463-6417 online (C) 2001 Taylor \& Francis Ltd 
state and the lowest excited level. If the spectrum is gapless, one can still define $\Delta$ as a pseudogap, splitting the lower-energy and the higher-energy parts in the density fluctuation spectrum. Generally speaking, $\Delta$ vanishes at the transition which occurs at a critical value $g_{\mathrm{c}}$ of a dimensionless parameter $g$ :

$$
\Delta \propto\left|g-g_{\mathrm{c}}\right|^{2 \nu},
$$

where $z \nu$ is a critical exponent which takes a universal value independent of the microscopic details of the Hamiltonian describing the system. We shall denote by $\Delta_{+}$and $\Delta_{-}$the values of the gap above and below $g_{c}$ respectively. Apart from an eventual constant of proportionality, both quantities are characterized by the same critical exponent $z \nu$.

The other scale is provided by the correlation length $\xi$. It is defined as the length scale characterizing the exponential decay of the equal-time correlation function $\langle M(\mathbf{r}, t) M(\mathbf{0}, t)\rangle$ in the disordered phase at $T=0$. The length scale $\xi$ diverges at the quantum critical point defined at $T=0$ and $g=g_{\mathrm{c}}$ according to

$$
\xi^{-1} \propto\left|g-g_{\mathrm{c}}\right|^{\nu} .
$$

Putting together the disappearance of $\Delta$ and the divergence of $\xi$ at the transition, one can write

$$
\Delta \propto \xi^{-z},
$$

where $z$ is the dynamic exponent. Those critical exponents are all defined at $T=0$ and $g \rightarrow g_{\mathrm{c}}$. However, they are also relevant at finite temperatures and $g \neq g_{\mathrm{c}}$.

Figure 1 displays the schematic phase diagram $(T, g)$ that can be derived. The solid curve is the line of phase transitions separating the long-range-ordered and the

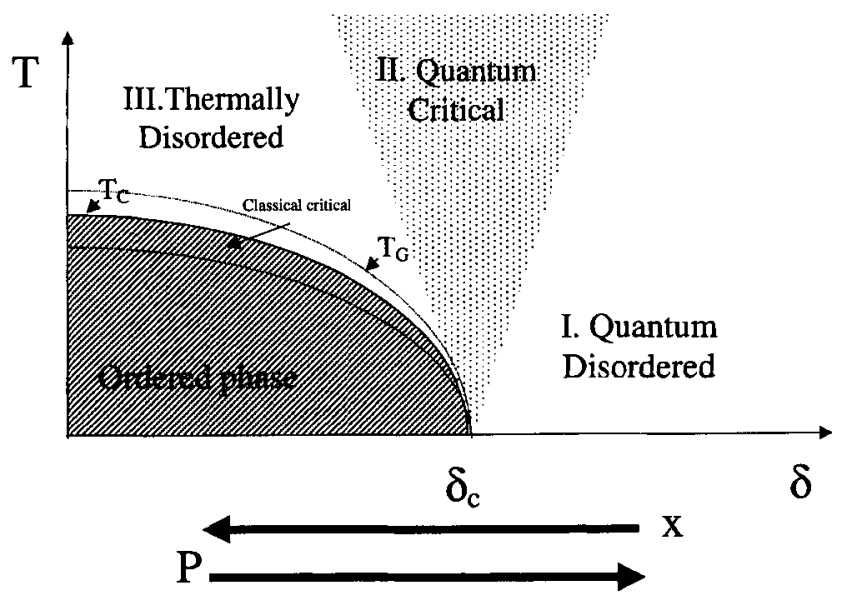

Figure 1. Schematic phase diagram as a function of temperature $T$ and dimensionless coupling $g$. The solid curve $T_{\mathrm{c}}$ is the line of phase transitions separating the long-range ordered and disordered phases. The upper dotted line is the Ginzburg temperature $T_{\mathrm{G}}$. An effective classical theory applies in the region located between the two dotted curves surrounding $T_{\mathrm{c}}$. Regime I is the quantum disordered regime characterized by quantum fluctuations. Regime III is the thermally disordered regime in which all the physical quantities are driven by temperature only. Regime II is the intermediate quantum critical regime in which both quantum and classical fluctuations are relevant. The arrows below the graph indicate how $g$ can be tuned by a chemical concentration change $x$ or pressure $P$. 
disordered phases. The dotted curve corresponds to the Ginzburg temperature $T_{\mathrm{G}}$ below which classical fluctuations are important. In the classical critical region, an effective classical theory applies. Regime $\mathrm{I}$ is the quantum disordered regime driven by quantum fluctuations as resulting from the Heisenberg uncertainty principle. In contrast, only thermal fluctuations are relevant in regime III above $T_{G}$. Finally, regime II is the quantum critical regime characterized by a rich interplay of effects driven by both quantum and thermal fluctuations.

\section{§2. EXPERIMENTAL EXAMPLES}

We shall mention some recent experimental studies of second-order quantum phase transitions.

\section{1. $\mathrm{LiHoF}_{4}$}

In the insulator $\mathrm{LiHoF}_{4}$, the $\mathrm{Ho}$ ions fluctuate between two spin states depending on whether the spins are parallel or antiparallel to a particular crystalline axis. At zero temperature, applying an external magnetic field perpendicular to the magnetic axis makes the system go from a ferromagnetic ground state to a quantum paramagnetic state in which long-range ferromagnetic order is destroyed owing to quantum tunnelling between the two spin states (Bitko et al. 1996). The quantum critical point is reached at a critical value $H_{C}$ of the magnetic field. Raising the temperature also destroys the long-range magnetic order driving the system to a high-temperature magnetically disordered state. Note that, in the latter state, the thermal paramagnet is fundamentally different in nature from the quantum paramagnet formed at zero temperature.

\subsection{Heavy-fermion compounds}

One of the most striking properties of heavy-fermion compounds discovered in the last few years is the existence of a quantum phase transition driven by change in chemical composition, pressure or magnetic field. For example, $\mathrm{CeCu}_{6-x} \mathrm{Au}_{x}$ (von Lohneysen et al. 1994, von Lohneysen 1996) and $\mathrm{Ce}_{1-x} \mathrm{La}_{x} \mathrm{Ru}_{2} \mathrm{Si}_{2}$ (Kambe et al. 1996, Raymond et al. 1997) present a transition from a magnetically disordered phase to a long-range antiferromagnetic order at $x_{\mathrm{c}}=0.1$ and $x_{\mathrm{c}}=0.08$ respectively. Other systems such as $\mathrm{CeIn}_{3}, \mathrm{CePd}_{2} \mathrm{Si}_{2}$ (Mathur et al. 1998), $\mathrm{CeNi}_{2} \mathrm{Ge}_{2}$ (Steglich et al. 1996) and $\mathrm{U}_{1-x} \mathrm{Y}_{x} \mathrm{Pd}_{3}$ (Seaman et al. 1991) exhibit the same behaviour. Once a long-range magnetic order is set up, the effect of a pressure or a magnetic field is to drive the system back to a magnetically disordered phase. Remarkably, while far from the quantum critical point the magnetically disordered phase is a Fermi liquid with large effective masses associated with the formation of heavy quasiparticles, there are some indications that the thermal properties of the system in the disordered phase close to the quantum critical point differ from that of a Fermi liquid (Coleman et al. 1996, Coleman 1999). Typically in $\mathrm{CeCu}_{5.9} \mathrm{Au}_{0.1}$ (von Lohneysen et al. 1994, von Lohneysen 1996), the specific heat $C$ depends on $T$ as $C / T \propto-\ln \left(T / T_{0}\right)$, the magnetic susceptibility as $\chi \propto 1-\alpha T^{1 / 2}$, and the $T$-dependent part of the resistivity as $\Delta \rho \propto T$ instead of $C / T \propto \chi \propto$ constant and $\Delta \rho \propto T^{2}$ in the Fermi-liquid state. The origin of this non-Fermi-liquid behaviour is presently a problem of considerable debate.

An important element in the knowledge of the quantum critical point has been recently provided by inelastic neutron scattering (INS) experiments performed on a $\mathrm{CeCu}_{5.9} \mathrm{Au}_{0.1}$ single crystal (Schröder et al. 1998, 2000). The dynamical spin suscept- 
ibility $\chi^{\prime \prime}(\mathbf{q}, \omega)$ near the magnetic instability wave-vector $\mathbf{Q}$ has been found to obey an anomalous $\omega / T$ scaling law as a function of temperature:

$$
\chi^{\prime \prime}(\mathbf{Q}, \omega) \propto T^{-\alpha} g\left(\frac{\omega}{T}\right),
$$

with $\alpha$ of the order of 0.75 . Moreover, these $\omega$ and $T$ dependences occur over the entire Brillouin zone and are revealed in the bulk susceptibility too. This strongly suggests that the spin dynamics are critical not only on large length scales but also on atomic length scales, contrary to what happens in the traditional itinerant magnetism picture. We shall return to this point at the end of the paper.

Let us focus now on the general problem of quantum phase transitions between a Fermi liquid and a magnetically ordered phase. This is still a largely controversial topic in which many aspects are not fully understood. Details of the topology of the Fermi surface often means introducing important nesting effects and Kohn anomalies in the spin susceptibility when the magnetic wave-vector spans the Fermi surface. For simplicity, we shall not consider those nesting effects.

The rest of the paper is organized as follows. In $\S 3$, we show how one can integrate out the fermion fields to derive an effective bosonic model associated with the fluctuations in the ordering fields. The resulting effective action exhibits a dynamic exponent $z$ equal to 3 in the vicinity of a ferromagnetic critical point and 2 for the antiferromagnetic case. In $\S 4$, we give simple arguments based on an expansion to first order in the effective interaction $u$ to determine an upper critical dimension $D_{\mathrm{c}}=4$ (where $D=d+z$ and $d$ is the spatial dimension) below which first-order fluctuations diverge and the mean-field description is no longer valid. An alternative tricritical crossover approach valid at $D<D_{\mathrm{c}}$ in the large- $N$ limit is described in $\S 5$. $\S 6$ gives a short presentation of perturbative renormalization group (RG) approaches and their links to self-consistent renormalized spin fluctuation (SCRSF) theories to understand the quantum-classical crossover in the vicinity of the quantum critical point. In $\S 7$ the approach is generalized to the Kondo lattice model which is believed to describe the heavy-fermion situation. Finally, we quote some recent INS experiments performed on heavy fermions leading to an unusual scaling law in $\omega / T$ for the dynamical spin susceptibility, which reveals critical local modes beyond the itinerant magnetism picture inherent to perturbative $R G$ and SCRSF approaches. We mention new attempts to describe this local quantum critical point.

\section{§3. DeRivation OF AN EFFECTIVE Bosonic THEORY}

We start from the Hubbard Hamiltonian which represents the archetypal model for correlated electron system. For a single non-degenerate band of electrons of spin $\frac{1}{2}$, the Hamiltonian is

$$
H=\sum_{k \sigma} \varepsilon_{k} c_{k \sigma}^{\dagger} c_{k \sigma}+U \sum_{i} n_{i \uparrow} n_{i \downarrow}
$$

where the last term is the Coulomb repulsion term expressed in the Wannier representation, $\varepsilon_{k}=-\left[\sum_{\delta} t_{i j} \exp (\mathbf{i k} \cdot \boldsymbol{\delta})\right] / z, t_{i j}$ are the overlap integrals between neighbouring sites and $z$ is the coordination number. The Hamiltonian is characterized by competition between the kinetic term and the Coulomb interaction $U$, which generally induces a quantum phase transition from a Fermi liquid to a magnetically ordered state at a critical value of $U / t$. 
By integrating out the fermion fields, we shall first show how from the Hubbard Hamiltonian we can derive an effective bosonic theory in terms of the fluctuations of the ordering fields $\Phi_{i}$ associated with the magnetizations. Following Hertz and Klenin (1974), we divide the proof into the following steps. The calculations are performed in the magnetically disordered phase.

First let us write the Coulomb term as a function of charge and spin density variables:

$$
U \sum_{i} n_{i \uparrow} n_{i\rfloor}=\frac{U}{4} \sum_{i}\left(n_{i \uparrow}+n_{i \downarrow}\right)^{2}-\frac{U}{4} \sum_{i}\left(n_{i \uparrow}-n_{i \downarrow}\right)^{2},
$$

where $\left(n_{i \uparrow}-n_{i \downarrow}\right) / 2=\Phi_{i}^{z}$ is the $z$ magnetization. Note that one can extend equation (6) to all three directions $x, y$ and $z$ in order to preserve the spin rotation invariance.

Let us consider the functional integral of the partition function

$$
\begin{aligned}
Z & =\int \mathcal{D} c_{i \sigma} \exp \left[-\int_{0}^{\beta} \mathcal{L}(\tau) \mathrm{d} \tau\right], \\
\mathcal{L}(\tau) & =\sum_{i \sigma} c_{i \sigma}^{\dagger} \partial_{\tau} c_{i \sigma}+H(\tau),
\end{aligned}
$$

and perform a Hubbard-Stratonovich transformation on the Coulomb interaction term. Using the identity

$$
\int \mathrm{d} \Phi_{i}^{z}(\tau) \exp \left(-\int_{0}^{\beta}\left[\Phi_{i}^{z}(\tau)-U^{1 / 2} c_{i \sigma_{1}}^{\dagger} \tau_{\sigma_{1} \sigma_{2}}^{z} c_{i \sigma_{2}}\right]\left[\Phi_{i}^{z}(\tau)-U^{1 / 2} c_{i \sigma_{3}}^{\dagger} \tau_{\sigma_{3} \sigma_{4}}^{z} c_{i \sigma_{4}}\right] \mathrm{d} \tau\right)=1
$$

in which the summation on the spin indices $\sigma_{i}$ are implicit and $\tau^{i}$ represent the Pauli matrices, we find that

$$
Z=Z_{0} \iint \mathcal{D} c_{i \sigma} \mathrm{d} \Phi_{i}^{z}(\tau) \exp \left(-\int_{0}^{\beta}\left[\Phi_{i}^{z}(\tau) \Phi_{i}^{z}(\tau)-U^{1 / 2} \Phi_{i}^{z}(\tau) c_{i \sigma^{\dagger}}^{\dagger} \tau_{\sigma \sigma^{\prime}}^{z} c_{i \sigma^{\prime}} \mathrm{d} \tau\right) .\right.
$$

Integrating out the grassmannian variables $c_{i \sigma}$, we obtain

$$
\begin{gathered}
Z=Z_{0} \int \mathrm{d} \Phi_{i}^{z}(\tau) \exp \left[-S_{\mathrm{eff}}\left(\Phi_{i}\right)\right] \\
S_{\mathrm{eff}}\left(\Phi_{i}\right)=\int_{0}^{\beta} \int_{0}^{\beta}\left(\Phi_{i}^{z}(\tau) \Phi_{i}^{z}(\tau) \delta\left(\tau-\tau^{\prime}\right)-\operatorname{Tr}\left\{\ln \left[1-U^{1 / 2} \Phi_{i}(\tau) G_{0}^{i j}\left(\tau-\tau^{\prime}\right)\right]\right\}\right) \mathrm{d} \tau \mathrm{d} \tau^{\prime}
\end{gathered}
$$

where $G_{0}$ is the bare Green function of electrons at $U=0$.

Expanding the $\operatorname{Tr}\{\ln []$.$\} term up to the second and fourth order in \Phi$, we have

$$
\begin{aligned}
S_{\mathrm{eff}}\left(\Phi_{i}\right)= & \beta V \sum_{q, \mathrm{i} \omega_{\nu}}\left[1-U \chi_{0}\left(q, \mathrm{i} \omega_{\nu}\right)\right] \Phi\left(q, \mathrm{i} \omega_{\nu}\right) \Phi\left(-q,-\mathrm{i} \omega_{\nu}\right) \\
& +u \beta V^{4} \sum_{q_{i}, \mathrm{i} \omega_{i}} \Phi\left(q_{1}, \mathrm{i} \omega_{1}\right) \Phi\left(q_{2}, \mathrm{i} \omega_{2}\right) \Phi\left(q_{3}, \mathrm{i} \omega_{3}\right) \\
& \times \Phi\left(-q_{1}-q_{2}-q_{3},-\mathrm{i} \omega_{1}-\mathrm{i} \omega_{2}-\mathrm{i} \omega_{3}\right),
\end{aligned}
$$

where $\chi_{0}\left(q, i \omega_{\nu}\right)$ is the bare dynamical susceptibility and $u$ an effective interaction assumed to be local. 
Close to a ferromagnetic phase transition, we can use the Lindhard expansion of $\chi_{0}(q, \omega)$ around $q=\omega=0$ :

$$
\chi_{0}\left(q, \mathrm{i} \omega_{\nu}\right)=\chi_{0}(0,0)-b \frac{q^{2}}{k_{F}^{2}}+\mathrm{i} a \frac{\omega}{\Gamma_{q}}
$$

where $k_{\mathrm{F}}$ is the Fermi wave-vector, $a$ and $b$ are constants and $\Gamma_{q}=q v_{F}$ is the relaxation rate which vanishes in the $q \rightarrow 0$ limit. Note that this disappearance is imposed by some symmetry arguments because the fluctuations of the order parameter are conserved in the ferromagnetic case. Hence one can obtain the effective bosonic action in the ferromagnetic case:

$$
\begin{aligned}
S_{\mathrm{eff}}\left(\Phi_{i}\right)= & \beta V \sum_{q, \mathrm{i} \omega_{\nu}}\left(\delta+q^{2}+\frac{\left|\omega_{\nu}\right|}{q}\right) \Phi\left(q, \mathrm{i} \omega_{\nu}\right) \Phi\left(-q,-\mathrm{i} \omega_{\nu}\right) \\
& +u \beta V^{4} \sum_{q_{i}, \mathrm{i} \omega_{i}} \Phi\left(q_{1}, \mathrm{i} \omega_{1}\right) \Phi\left(q_{2}, \mathrm{i} \omega_{2}\right) \Phi\left(q_{3}, \mathrm{i} \omega_{3}\right) \\
& \times \Phi\left(-q_{1}-q_{2}-q_{3},-\mathrm{i} \omega_{1}-\mathrm{i} \omega_{2}-\mathrm{i} \omega_{3}\right)
\end{aligned}
$$

where $\delta=1-U \chi_{0}\left(q, i \omega_{\nu}\right)$ called the Stoner factor measures the distance to the magnetic instability.

The result can be generalized to the case of an antiferromagnetic instability. In this case, if we note $q$ the deviation from the antiferromagnetic wave-vector $\mathbf{Q}=(\pi, \pi, \pi)$, the expansion expressed in equation (12) is still valid around $\mathbf{Q}$. However, since the fluctuations are not conserved in the antiferromagnetic case, the relaxation rate $\Gamma_{q}$ is now $q$ independent. The first term of equation (13) is modified in the following way:

$$
S_{\text {eff }}^{(2)}\left(\Phi_{i}\right)=\beta V \sum_{q, i \omega_{\prime \prime}^{\prime}}\left(\delta+q^{2}+\left|\omega_{\nu}\right|\right) \Phi\left(q, i \omega_{\nu}\right) \Phi\left(-q,-\mathrm{i} \omega_{\nu}\right)
$$

The justification of the expansion of the $\operatorname{Tr}\{\ln [\cdot]\}$ term in equation (10) up to the second and fourth order in $\Phi$ lies in the fact that the $\Phi_{i}$ representing the fluctuations of the magnetization are expected to be small in the magnetically disordered phase close to the quantum critical point. However, the expansion would not stand within the long-range ordered state where the determination of the finite magnetization $M_{0}$ which $\Phi$ can be expanded about $\left(\Phi=M_{0}+\delta \Phi\right)$ requires minimization of the full expression of the free energy including all orders and not stopping to the fourth order. This is precisely what the equivalent of the gap equation for the magnetization does.

To end this section, we mention that recently, this procedure of integrating out the fermionic degrees of freedom has been questioned (Kirkpatrick and Belitz 1996, Vojta et al. 1997). These authors showed that keeping electronic correlations gives rise to an effective long-range interaction among the order parameter fluctuations. They emphasized that the presence of the resulting wave-vector-dependent nonanalyticities may introduce qualitative changes in the description of the phase transition. 


\section{§4. Perturbation theory in $u$ : existence of AN UPPer CRitiCal Dimension}

In the effective bosonic theory presented in the previous section, $q$ and $\omega$ do not appear at the same order. They may do so in some different models. For convenience in this section, we shall only consider the case when $\omega$ appears at the second order too and use the relativistic notation $Q^{2}=q^{2}+\omega^{2}$. We shall show the upper critical dimension to be equal to 4 for $D=d+1$ as a result of diverging critical fluctuations when $D<4$. The result will be generalized later when a dynamic exponent $z$ instead of 1 makes $D$ change to $d+z$ keeping the same value of 4 for the upper critical dimension. In realistic notation, the effective action can be written

$$
\begin{aligned}
S_{\mathrm{cff}}\left(\Phi_{i}\right)= & \beta V \sum_{q, \mathrm{i} \omega_{\nu^{\prime}}}\left(\delta+Q^{2}\right) \Phi(Q) \Phi(-Q) \\
& +u \beta V^{4} \sum_{q_{i}, \mathrm{i} \omega_{i}} \Phi\left(Q_{1}\right) \Phi\left(Q_{2}\right) \Phi\left(Q_{3}\right) \Phi\left(-Q_{1}-Q_{2}-Q_{3}\right) .
\end{aligned}
$$

At zero temperature and to zeroth order in $u$, the spin susceptibility $\chi(Q)$ related to the correlation function in $\Phi$ is given by

$$
\left[\chi^{(0)}(Q)\right]^{-1}=Q^{2}+\delta \text {. }
$$

The uniform static susceptibility $\chi^{(0)}(0)$ diverges at $\delta_{\mathrm{c}}^{(0)}=0 . \delta$ plays the role of a tuning parameter and the quantum phase transition takes place at $\delta_{\mathrm{c}}^{(0)}=0$.

To first order in $u$, the result is changed in the following way:

$$
[\chi(Q)]^{-1}=Q^{2}+\delta+\frac{u}{2} \int \frac{\mathrm{d}^{D} Q}{(2 \pi)^{D}} \frac{1}{Q^{2}+\delta},
$$

where $D=d+1$ and $d$ is the spatial dimension. The critical value of $\delta$ is given by

$$
\delta_{\mathrm{c}}=-\frac{u}{2} \int \frac{\mathrm{d}^{D} Q}{(2 \pi)^{D}} \frac{1}{Q^{2}+\delta_{\mathrm{c}}},
$$

Denoting $s=\delta-\delta_{\mathrm{c}}$ which measures the deviation of the system from the quantum critical point,

$$
[\chi(Q)]^{-1}=Q^{2}+s+\frac{u}{2} \int \frac{\mathrm{d}^{D} Q}{(2 \pi)^{D}}\left(\frac{1}{Q^{2}+\delta}-\frac{1}{Q^{2}}\right) .
$$

For $D>4$, the integrand diverges in the $Q \rightarrow \infty$ limit and one needs to introduce a cut-off $\Lambda$ for $Q$. One can then perform an expansion in $s$ :

$$
[\chi(Q)]^{-1}=Q^{2}+s\left(1-c_{1} u \Lambda^{D-4}\right),
$$

where $c_{1}$ is a non-universal constant depending on the nature of the cut-off. $\chi(0)$ diverges in the $s \rightarrow 0$ limit. Mean-field critical properties still apply with small firstorder corrections.

For $D<4$, the integrand now converges in the $Q \rightarrow \infty$ limit. Therefore, under the condition $u \ll A^{D-4}$, one can put $A \rightarrow \infty$ in the integral. To first order in $u$,

$$
[\chi(Q)]^{-1}=Q^{2}+s\left(1-\frac{1}{2} \frac{2 \Gamma[(4-D) / 2]}{(D-2)(4 \pi)^{D / 2}} \frac{u}{s^{(4-D) / 2}}\right) .
$$

Although $u$ is small, the correction to the mean-field result is important. It even diverges in the $s \rightarrow 0$ limit. One can then deduce an upper critical value for the 
dimension $D_{\mathrm{c}}=4$. At $D<D_{\mathrm{c}}$ the mean-field results are not correct and a more sophisticated resummation of the perturbation expansion is required. For this purpose, we now present a large- $N$ theory, introducing a tricritical crossover function which allows us to do that.

\section{$\S 5$. LARGE- $N$ THEORY AND TRICRITICAL CROSSOVERS}

For $D<4$, equation (21) established at first order in $u$ suggests that $[\chi(Q)]^{-1}$ can be expressed as

$$
[\chi(Q)]^{-1}=s \psi_{D}(q, v)
$$

where $q=Q / s^{1 / 2}$ and $v=u / s^{(4-D) / 2}$ and where we have introduced $\psi_{D}[q, v]$ as a universal function called the tricritical crossover function (Brézin and Zinn-Justin 1985, Miranda et al. 1997, Sachdev 1999). To first order in $u, \psi_{D}(q, v)$ can be identified with

$$
\psi_{D}(q, v)=q^{2}+1-\frac{\Gamma[(4-D) / 2]}{(D-2)(4 \pi)^{D / 2}} v+\mathrm{O}\left(v^{2}\right) .
$$

If we assume that we can put $\Lambda \rightarrow \infty$ in all the higher-order terms in $u$, we can expect $[\chi(Q)]^{-1}$ to take the form expressed in equation (25).

We now explain how, in the large- $N$ limit, $\psi_{D}(q, v)$ can be determined at any order in $v$. To do that, we first extend the previous descriptions to any value of the degeneracy of the Hubbard-Stratonovich parameter $\Phi_{\alpha}, \alpha=1,2, \ldots, N$ and let $N$ go to infinity at the end of the calculation. Performing a Hubbard-Stratonovich transformation on the the $\Phi^{4}$ term, one obtains in the large- $N$ limit

$$
[\chi(Q)]^{-1}=Q^{2}+\delta+u \frac{N+2}{6}\left\langle\Phi^{2}\right\rangle
$$

where $\left\langle\Phi^{2}\right\rangle$ is self-consistently determined as in the self-consistent one-loop approximation according to

$$
\left\langle\Phi^{2}\right\rangle=\int \frac{\mathrm{d}^{D} Q}{(2 \pi)^{D}} \frac{1}{Q^{2}+\delta+u[(N+2) / 6]\left\langle\Phi^{2}\right\rangle} .
$$

Hence $\delta_{c}$ is defined by

$$
\delta_{\mathrm{c}}+\left.u \frac{N+2}{6}\left\langle\Phi^{2}\right\rangle\right|_{\delta=\delta_{\mathrm{c}}}=0
$$

and

$$
[\chi(Q)]^{-1}=Q^{2}+\left(\delta-\delta_{c}\right)+u \frac{N+2}{6}\left[\left\langle\Phi^{2}\right\rangle-\left.\left\langle\Phi^{2}\right\rangle\right|_{\delta=\delta_{c}}\right] .
$$

The critical crossover function $\psi_{D}(q, v)$ can be identified with

$$
\psi_{D}(q, v)=q^{2}+\pi_{D}(v),
$$

where the function $\pi_{D}(v)$ is the solution of the following nonlinear equation:

$$
\pi_{D}(v)+N v \frac{\Gamma[(4-D) / 2]}{3(D-2)(4 \pi)^{D / 2}}\left[\pi_{D}(v)\right]^{(D-2) / 2}=1 .
$$


In the $v \rightarrow \infty$ limit, $\pi_{D}(v) \propto v^{-2 /(D-2)}$ and $[\chi(0)]^{-1}$ behaves as $s^{D /(D-2)}$ at small $s$. The latter result on $[\chi(0)]^{-1}$ settles the difficulties arising from the diverging correction term obtained to first order in $u$ when $D<D_{\mathrm{c}}$.

\section{§6. Perturbative renormalization group}

We refer the reader to a number of very good reviews existing in the literature on this sophisticated and powerful approach. We shall simply try to clarify the essential points involved in the method. Let us start again from the effective action derived in $\S 4$ :

$$
\begin{aligned}
S_{e f f}(\Phi)= & \frac{1}{2} \sum_{q, \omega}\left(\delta+q^{2}+\frac{|\omega|}{q}\right)|\Phi(q, \omega)|^{2} \\
& +\frac{1}{2} u \frac{1}{\beta \mathcal{N}} \sum_{q_{i}, \omega_{i}} \Phi\left(q_{1}, \omega_{1}\right) \Phi\left(q_{2}, \omega_{2}\right) \Phi\left(q_{3}, \omega_{3}\right) \\
& \times \Phi\left(-q_{1}-q_{2}-q_{3},-\omega_{1}-\omega_{2}-\omega_{3}\right),
\end{aligned}
$$

where $\mathcal{N}$ is the number of sites and $\beta$ is the inverse temperature. The general idea of the RG approach is to eliminate the short-range and short-time details of the fluctuations of the order parameter to derive a renormalized effective action in which the different parameters are rescaled. As shown in figure 2, the aim is then to eliminate the contribution to $\Phi(q, \omega)$ from the outer shell with large values of $q$ and $\omega$. We also define the complementary inner shell characterized by small values of $q$ and $\omega$. Following Hertz' (1976) paper, we show how to derive the scaling equations in two steps: first keep the inner-shell contribution only, and then add the remaining contribution from the outer shell. This provides us with two scaling equations for $\delta$ and $u$ : the Stoner factor and the effective interaction respectively. Later we shall mention how Millis showed that it is crucial to consider an additional scaling equation for temperature.

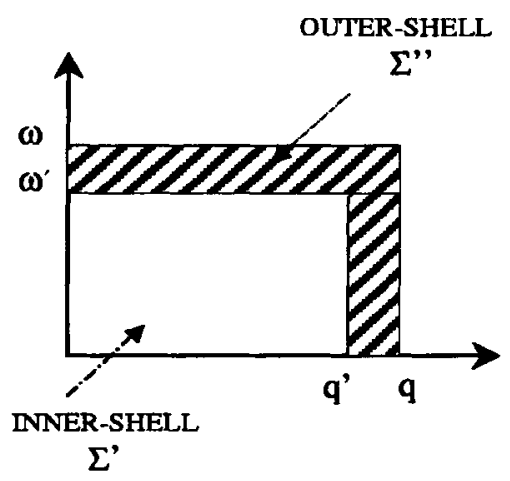

Figure 2. Illustration of the scaling procedure in $q$ and $\omega$. The shaded region represents the outer-shell contribution associated with the sum $\Sigma^{\prime \prime}$ over the $q$ and $\omega$ variables. The complementary inner-shell contribution corresponds to the sum $\Sigma^{\prime}$ over $q$ and $\omega$. 


\subsection{Elimination of the outer-shell contribution within the inner shell contribution} to $S_{\mathrm{eff}}$

First, let us remove the outer-shell contribution to $\Phi(q, \omega)$. Since $q$ and $\omega$ appear in the quadratic term of $S_{\text {eff }}(\Phi)$ at different orders, an anisotropic scaling procedure is required. The outer-shell contribution is defined by

$$
\begin{aligned}
\exp (-l) & <q<1, \\
\exp (-z l) & <\omega<1,
\end{aligned}
$$

where $l$ is infinitesimal and $z$ will be proved later to coincide with the dynamic exponent. Retaining the contribution to $S_{\text {eff }}(\Phi)$ from the inner shell only with small $q$ and $\omega$ values, one obtains the second order

$$
S_{\text {eff }}^{(2)}(\Phi)=\frac{1}{2} \beta \mathcal{N} \int_{0}^{\exp (-l)} \frac{\mathrm{d}^{d} q}{(2 \pi)^{d}} \int_{0}^{\exp (-z l)} \frac{\mathrm{d} \omega}{2 \pi}\left(\delta^{\prime}+q^{2}+\frac{|\omega|}{q}\right)|\Phi(\mathrm{q}, \omega)|^{2} .
$$

The change from $\delta$ to $\delta^{\prime}$ is of the order of $l$.

The next step consists in rescaling the variables $q$ and $\omega$ in the following way:

$$
\begin{aligned}
& q^{\prime}=q \exp (l), \\
& \omega^{\prime}=\omega \exp (z l) .
\end{aligned}
$$

With this change in variables, we have

$$
\begin{aligned}
S_{\text {eff }}^{(2)}(\Phi)= & \frac{1}{2} \beta \mathcal{N} \exp [-(d+z) l] \int_{0}^{l} \frac{\mathrm{d}^{d} q^{\prime}}{(2 \pi)^{d}} \int_{0}^{1} \frac{\mathrm{d} \omega^{\prime}}{2 \pi}\left(\delta^{\prime}+q^{\prime 2} \exp (-2 l)+\frac{\left|\omega^{\prime}\right|}{q^{\prime} \exp [-(z-1) l]}\right) \\
& \times\left|\Phi\left(q^{\prime} \exp (-l), \omega^{\prime} \exp (-z l)\right)\right|^{2} .
\end{aligned}
$$

The fields $\Phi(q, \omega)$ are then rescaled so that the terms in $q^{\prime 2}$ and $\left|\omega^{\prime}\right| / q^{\prime}$ in $S_{\text {eff }}^{(2)}(\Phi)$ are unchanged. Because of the anisotropic scaling procedure introduced earlier, we are allowed to do that as soon as we choose $z$ equal to the dynamic exponent 3 in the ferromagnetic case. Introducing the scaling

$$
\Phi^{\prime}\left(q^{\prime}, \omega^{\prime}\right)=\Phi(q, \omega) \exp \left(\frac{-(d+z+2) l}{2}\right)
$$

one obtains

$$
S_{\mathrm{eff}}^{(2)}(\Phi)=\frac{1}{2} \beta \mathcal{N} \int_{0}^{1} \frac{\mathrm{d}^{d} q^{\prime}}{(2 \pi)^{d}} \int_{0}^{1} \frac{\mathrm{d} \omega}{2 \pi}\left(\exp (2 l) \delta^{\prime}+q^{\prime 2}+\frac{\left|\omega^{\prime}\right|}{q^{\prime}}\right)\left|\Phi\left(q^{\prime}, \omega^{\prime}\right)\right|^{2} .
$$

We can see from this expression that the initial form of $S_{\text {eff }}^{(2)}(\Phi)$ is recovered provided that $\exp (2 l) \delta^{\prime}=\delta$. This gives us the first part of the scaling equation for $\delta$ :

$$
\frac{\mathrm{d} \delta}{\mathrm{d} l}=2 \delta
$$

In the same way, the effective action to the fourth order in $u$ can be expressed as

$$
\begin{aligned}
S_{\mathrm{eff}}^{(4)}(\Phi)= & \frac{1}{4}(\beta \mathcal{N})^{2} u^{\prime} \exp [-3(d+z) l] \exp \left(\frac{4(d+z+2) l}{2}\right) \int_{0}^{1} \frac{\mathrm{d}^{d} q_{i}{ }^{\prime}}{(2 \pi)^{d}} \int_{0}^{1} \frac{\mathrm{d} \omega_{i}}{2 \pi} \\
& \times \Phi\left(q_{1}^{\prime}, \omega_{1}^{\prime}\right) \Phi\left(q_{2}^{\prime}, \omega_{2}^{\prime}\right) \Phi\left(q_{3}^{\prime}, \omega_{3}^{\prime}\right) \Phi\left(-q_{1}^{\prime}-q_{2}^{\prime}-q_{3}^{\prime},-\omega_{1}^{\prime}-\omega_{2}^{\prime}-\omega_{3}^{\prime}\right),
\end{aligned}
$$


where the summation $\prod_{i=1,2,3}$ on the subscript $i$ is implicit. In order to keep the quartic term unchanged, one needs to transform $u$ according to

$$
u^{\prime} \exp (\varepsilon l)=u
$$

with $\varepsilon=4-(d+z)$ enabling us to write the second scaling equation for $u$ :

$$
\frac{\mathrm{d} u}{\mathrm{~d} l}=\varepsilon u
$$

As for $\delta$, we shall show in $\S 6.2$ that the latter scaling equation should include a second term on the right-hand side to be complete. Before concluding this section, let us point out that the scaling equation (40) provides an alternative way to define the upper critical dimension for $D=d+z$ generalizing to any $z$ the result previously obtained in $\S 4$. The spatial dimension is increased by the dynamic exponent $z$ equal to 3 in the ferromagnetic case and to 2 in the antiferromagnetic case. Above the upper critical dimension found to be $D_{\mathrm{c}}=4, \varepsilon$ has a negative sign and $u$ is rescaled to zero. We are then left with the quadratic term of $S_{\text {eff }}$ only and the system reaches a Gaussian fixed point. In contrast, below $D_{\mathrm{c}}=4, \varepsilon$ has a positive sign. The interaction is relevant and a non-Gaussian fixed point is reached.

6.2. Incorporation of the remaining contribution from the outer shell to $S_{\mathrm{eff}}$

The last step consists in considering the missing contribution with large values of $q$ and $\omega$. If one denotes by $\sum_{q, \omega}^{\prime}$ and $\sum_{q, \omega}^{\prime \prime}$ the summation over the inner and the outer shells respectively, the corresponding correction to $S_{\mathrm{eff}}^{(4)}(\Phi)$ is

$$
\begin{aligned}
S_{\mathrm{eff}}^{(4)}(\Phi)= & \frac{u}{4 \beta \mathcal{N}} \sum_{q, \omega}{ }^{\prime \prime} \prod_{i=1}^{4} \Phi\left(q_{i}, \omega_{i}\right) \delta\left(\sum_{i=1}^{4} q_{i}\right) \delta\left(\sum_{i=1}^{4} \omega_{i}\right) \\
& +\frac{3 u}{2 \beta \mathcal{N}} \sum_{q, \omega}^{\prime \prime}|\Phi(q, \omega)|^{2} \sum_{q, \omega}^{\prime}|\Phi(q, \omega)|^{2} .
\end{aligned}
$$

The two terms on the right-hand side of the last equation correspond to the interaction of one particle in the outer shell with one particle in the outer shell and one particle in the inner shell respectively. Part of the action which is quadratic as $\sum_{q, \omega}^{\prime}|\Phi(q, \omega)|^{2}$ can be resummed. The final expression for $S_{\text {eff }}$ including all contributions from the inner and the outer shell up to the fourth order is

$$
\begin{aligned}
S_{\mathrm{eff}}^{(2)}(\Phi)= & \frac{1}{2} \sum_{q, \omega}^{\prime \prime}\left(\delta+q^{2}+\frac{|\omega|}{q}\right)|\Phi(q, \omega)|^{2} \\
& +\frac{u}{4 \beta \mathcal{N}} \sum_{q, \omega}^{\prime \prime} \prod_{i=1}^{4} \Phi\left(q_{i}, \omega_{i}\right) \delta\left(\sum_{i=1}^{4} q_{i}\right) \delta\left(\sum_{i=1}^{4} \omega_{i}\right) \\
& +\frac{1}{2} \sum_{q, \omega}^{\prime} \ln \left(\delta+q^{2}+\frac{|\omega|}{q}+\frac{3 u}{\beta N} \sum_{q, \omega}^{\prime \prime}|\Phi(q, \omega)|^{2}\right) .
\end{aligned}
$$

Expanding the $\ln (\cdot)$ term up to the fourth order in $\Phi(q, \omega)$ enables us to obtain the additional part of the scaling equations. Putting this together with the truncated part of the scaling equations obtained in $\S 6.1$, one can write the complete scaling equations for $\delta$ and $u$ : 


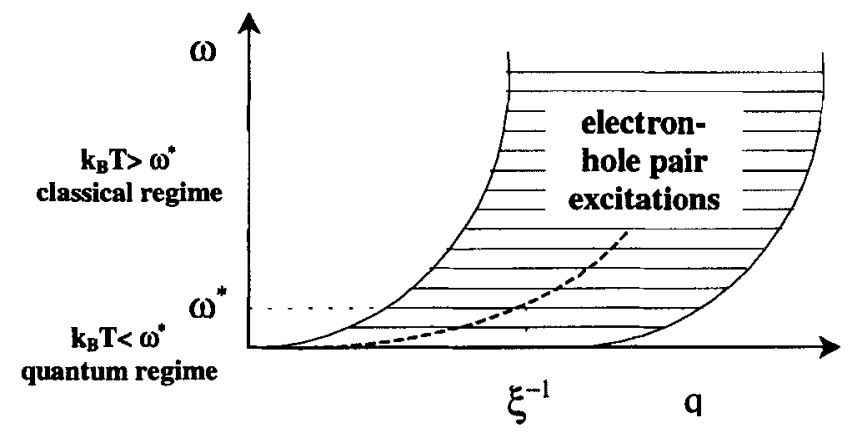

Figure 3. Continuum of electron-hole pair excitations. The broken curve represents the damped collective mode exhibiting a change in the dispersion at a characteristic energy $\omega^{*}$ and wave-vector $\xi^{-1} \propto \delta^{1 / 2}$. The energy scale $\omega^{*}$ defines the crossover temperature $T_{\mathrm{I}}$ separating the quantum from the classical regime.

$$
\begin{aligned}
& \frac{\mathrm{d} \delta}{\mathrm{d} l}=2 \delta+\frac{3 u}{\beta \mathcal{N}} \sum_{q, \omega}\left(\delta+q^{2}+\frac{|\omega|}{q}\right)^{-1}, \\
& \frac{\mathrm{d} u}{\mathrm{~d} l}=\varepsilon u-\frac{9 u^{2}}{\beta \mathcal{N}} \sum_{q, \omega}\left(\delta+q^{2}+\frac{|\omega|}{q}\right)^{-2} .
\end{aligned}
$$

These two equations constitute the whole set of RG equations derived by Hertz. The crossover temperature $T_{1}$ separating the quantum and the classical regimes is then defined in the following way. At the end of the scaling procedure, the quantum regime is reached if only the $\nu=0$ term contributes to the Matsubara frequency sum in equations (43) and (44). Later, Millis (1993) corrected Hertz' paper and showed that the temperature should be rescaled as well as $\delta$ and $u$, adding a third RG equation for temperature:

$$
\frac{\mathrm{d} T}{\mathrm{~d} l}=z T
$$

Then the criterion used to differentiate the quantum from the classical regime is slightly different. It depends on how the temperature $T^{*}$ at the end of the scaling procedure compares with an energy cut-off $\omega^{*}$.

We shall now give a physical interpretation to $T_{\mathrm{I}}$ in terms of the spectrum of spin excitations as schematized in figure 3. The shaded region corresponds to the Lindhard continuum of electron-hole pair excitations. It is defined by a finite value of the imaginary part of the bare dynamical susceptibility. The collective mode obtained from the poles of the inverse renormalized susceptibility is a damped magnon, as reported in figure 3. In the ferromagnetic case, the dispersion of the collective mode changes from a $q$ to a $q^{3}$ dependence at a characteristic value of the wave-vector $q^{*}=\xi^{-1} \propto \delta^{1 / 2}$. The energy scale $\omega^{*}$ corresponds to the energy of the collective mode on the scale of the magnetic length $\xi$. The regime is either quantum or classical depending on whether $T^{*}$ is found to be smaller or larger than $\omega^{*}$. The two regimes are characterized by different temperature behaviours of the physical quantities such as the magnetic correlation length $\xi$ and the coefficient $\gamma$ of the linear temperature dependence of the specific heat. Above a second cross- 


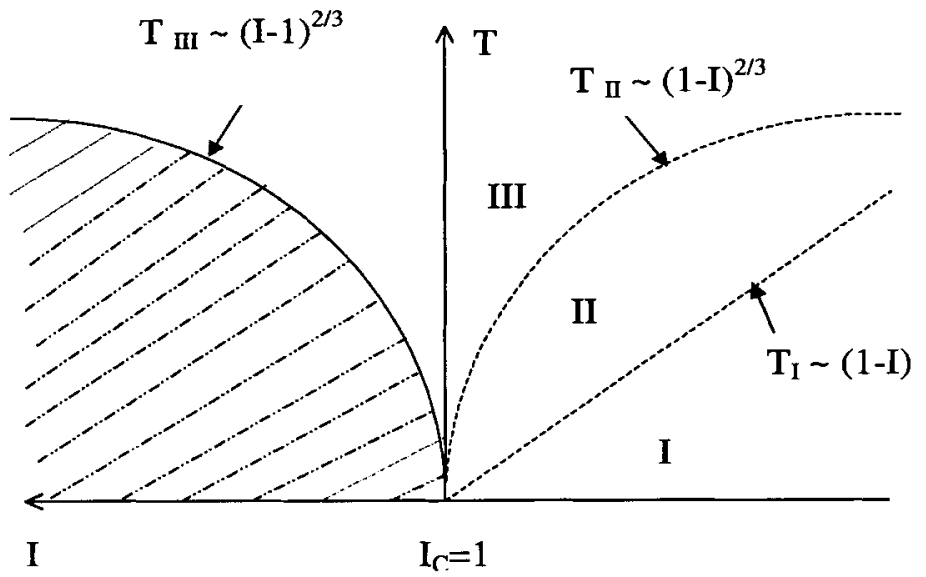

Figure 4. Phase diagram in the plane $(T, I)$ for dimension $d$ equal to 3 and $z=2$ for the Kondo lattice model (Lavagna and Pépin 2000). The shaded region represents the long-range antiferromagnetic phase bordered by the Neel temperature $T_{\mathrm{N}}$. The unshaded region marks the magnetically disordered regimes I, II and III associated with different behaviors of the system. Regime I is the quantum regime in which the energy of the relevant mode on the scale of $\xi$ is much greater than $k_{\mathrm{B}} T$. Regimes II and III are both classical regimes in which the thermal effects are important since the fluctuations on the scale of $\xi$ have energy much smaller than $k_{\mathrm{B}} T$. In regime II, $\xi$ is still controlled by $I-I$ but the staggered spin susceptibility is sensitive to the thermal fluctuations. In regime III, both $\xi$ and $\chi_{Q}^{\prime}$ are controlled by the temperature.

over temperature $T_{\mathrm{II}}$, all the relevant modes contributing to equations (43) and (44) have energies much less that $k_{\mathrm{B}} T$ and the physical quantities are driven by the temperature only. The results obtained by Millis are similar to those reported in figure 4 and depend on only the spatial dimension $d$ and the dynamic exponent $z$.

Note that an alternative approach to the magnetic phase transitions in the Hubbard model has been developed by Moriya and Kawabata (1973) and Moriya and Takimoto (1995) based on SCRSF theory. It leads to results very similar to those obtained by the perturbative RG method. Comparisons between the two approaches can be found in the paper by Millis (1993).

\section{$\S 7$. Heavy Fermions and the Kondo lattice model}

The model that is believed to describe the physics of heavy fermions is the Kondo lattice model where impurity spins distributed on the sites of a periodic lattice interact with the spin of local conduction electrons via a Kondo coupling $J_{\mathrm{K}}$. The main features of the model result from the competition between two energy scales (Doniach 1977): the Kondo temperature $T_{\mathrm{K}}$ corresponding to the binding energy at zero temperature of the singlet formed by the impurity spin screened by the spin of the conduction electrons; the Ruderman-Kittel-Kasuya-Yosida (RKKY) interactions between neighbouring spins mediated by the conduction electrons. The latter favours the formation of a long-range magnetic order while the former blocks it by Kondo screening. The consequence is the existence of a quantum critical point at a critical value of $J_{\mathrm{K}}$ below which a long-range magnetic order occurs. In the case 
when the total number of conduction electrons is less than one per site, all phases are in a metallic state.

The large- $N$ expansions (Auerbach and Levin 1986, Millis and Lee 1987) which have been carried out for these models (where $N$ represents the degeneracy simultaneously of the conduction electrons and of the spin channels) are known to give a good description of the Kondo effect but fail to account for the spin fluctuations since the RKKY interactions only appear at the order $1 / N^{2}$. With the aim of describing the critical phenomena around the quantum critical point of the heavyfermion systems, it has been recently proposed (Pépin and Lavagna 1999b, Lavagna and Pépin 2000) a self-consistent one-loop approximation for the $S=\frac{1}{2}$ Kondo lattice model $(N=2)$ that enlarges the standard 1/N expansion theories on the spin fluctuation effects in complete analogy with the SCRSF theory developed for the Hubbard model. It results in a quantum-classical crossover at a finite temperature depending on whether the temperature is lower or higher than the characteristic energy scale of the damped collective mode existing in the vicinity of the magnetic instability. We refer to paper by Lavagna and Pépin (2000) for a discussion of the related phase diagram at $d=2$ and $d=3$ with the predictions of a series of crossovers in the vicinity of the quantum critical point as reported in figure 4 .

\section{$\S 8$. Conclusion}

To conclude, we state that the perturbative RG approach and the related SCRSF theory, by eliminating the short-range details of the fluctuations of the order parameter, assume that the low-energy excitations are spatially extended and in essence do not take into account the critical local nature of the modes on the atomic length scale. As we mentioned at the end of $\$ 2$, there exist strong indications now, based on INS experiments on heavy fermions, that the magnetic excitations are critically local around the quantum critical point. This feature seems to be a characteristic of most of the strongly correlated electron systems including high- $T_{\mathrm{c}}$ superconductors. The existence of critical local modes is related to the formation of local moments in the ordered phase in contrast with the itinerant magnetism picture that is described by perturbative RG and SCRSF theories. Note that a scaling law in $\omega / T$ of the dynamical spin susceptibility has been obtained theoretically in the case of spin systems as for the random two-dimensional Heisenberg antiferromagnetic model (Sachdev and Ye 1992). There is an urgent need to develop theories to describe this type of local quantum critical point in itinerant systems. From this perspective, we shall mention two recent attempts to do this: on the one hand, calculations based on the dynamical mean-field theory (Si et al. 1999, 2000), which have recently lead to the scaling law in $\omega / T$ for the dynamical spin susceptibility in agreement with experimental observations; on the other hand supersymmetric approaches (Pépin and Lavagna 1997, 1999a, Coleman et al. 2000a,b) based on a mixed fermionic-bosonic representation of the spin, which has the advantage of capturing both the quasiparticle and the local moment features via the fermionic and bosonic degrees of freedom respectively.

\section{ACKNOWLEDGEMENTS}

I am grateful to Zsolt Gulácsi for giving me the opportunity to give this lecture. I would like to thank Nick Bernhoeft, Andrey Chubukov, Piers Coleman, Mucio Continentino, Jacques Flouquet, Gilbert Lonzarich, Catherine Pépin, Stéphane Raymond, Louis-Pierre Regnault, Almut Schröder and Hilbert von Löhneysen for helpful discussions. 


\section{REFERENCES}

AuerbaCh, A., and Levin, K., 1986, Phys. Rev. Lett., 57, 877.

Bitko, D., Rosenbaum, T. F., and Aeppli, G., 1996, Phys. Rev. Lett., 77, 940.

BréZin, E., and Zinn-Justin, J., 1985, Nucl. Phys. B, 257, 867.

Coleman, P., 1999, Physica B, 259-261, 353.

Coleman, P., Maple, B., and Millis, A. J. (editors), 1996, J. Phys.: condens. Matter, 8, and references therein.

Coleman, P., Pépin, C., and Tsvelik, A. M., 2000a, Phys. Rev. B, 62, 3852; 2000b, Nucl. Phys. B, 586, 641.

Doniach, S., 1977, Physica B, 91, 231.

Hertz, J. A., 1976, Phys. Rev. B, 14, 1165.

Hertz, J. A. and Klenin, M. A., 1974, Phys. Rev. B, 10, 1084.

Kambe, S., Raymond, S., Regnault, L. P., Flouquet, J., Lejay, P., and Haen, P., 1996, J. phys. Soc. Japan, 65, 3294.

Kirkpatrick, T. R., and Belitz, D., 1996, Phys. Rev. B, 53, 14364.

Lavagna, M., and Pépin, C., 1998, Acta Phys. Polonica B, 29, 3753; 2000, Phys. Rev. B, 62, 6450 .

Mathur, N. D., Grosche, F. M., Julian, S. R., Walker, I. R., Freye, D. M., Hasel wimmer, R. K. W., and LonZarich, G. G., 1998, Nature, 394, 39, and references therein.

Millis, A. J., 1993, Phys. Rev. B, 48, 7183.

Millis, A. J., and Lee, P. A., 1987, Phys. Rev. B, 35, 3394.

Miranda, E., Dobrosavljevic, V., and Kotliar, G., 1997, Phys. Rev. Lett., 78, 290.

Moriya, T., and Kawabata, A., 1973, J. phys. Soc. Japan, 34, 639.

MoriYa, T., and Tакimoto, T., 1995, J. phys. Soc. Japan, 64, 960.

Pépin, C., and Lavagna, M., 1997, Z. Phys. B, 103, 259; 1999a, Phys. Rev. B, 59, 2591; 1999b, ibid., 59, 12180.

Raymond, S., Regnault, L. P., Kambe, S., Mignod, J. M., Lejay, P., and Flouquet, J., 1997, J. low Temp. Phys., 109, 205.

SACHdev, S., 1999, Quantum Phase Transitions (Cambridge University Press).

SACHDEv, S., and YE, J., 1992, Phys. Rev. Lett., 69, 2411.

Schröder, A., Aeppli, G., Bucher, E., Ramazashyili, R., and Coleman, P., 1998, Phys. Rev. Lett., 80, 5623.

Schröder, A., Aeppli, G., Coldea, R., Adams, M., Stockert, O., von löhneysen, H., Bucher, E., Ramazashvili, R., and Coleman, P., 2000, Nature, 407, 351.

Seaman, C. L., Maple, M. B., Lee, B. W., Ghamaty, S., Torikachvili, M. S., Kang, J.-S., Liu, L.-Z., Allen, J., and Cox, D. L., 1991, Phys. Rev. Lett., 67, 2882.

Si, Q., Rabello, S., Ingersent, K., and SMiTh, J. L., 2000 , cond-mat/0011477.

Si, Q., Smith, J. L., and Ingersent, K., 1999, Int. J. mod. Phys. B, 13, 2331.

Steglich, F., Buschinger, B., Gegenwart, P., Lohmann, M., Helfrich, R., Langhammer, C., Hellman, P., Donnevert, L., Thomas, S., Link, A., Geiber, C., Lang, M., Sparn, G., and Assmus, W., 1996, J. Phys.: condens. Mater, 8, 9909.

Vojta, T., Belitz, D., Narayanan, R., and Kirkpatrick, T. R., 1997, Z. Phy's. B, 103, 451 .

von Löhneysen, H., 1996, J. Phys.: condens. Matter, 8, 9689, and references therein.

von Löhneysen, H., Schröder, A., Sieck, M., and Trappmann, T., 1994, Phys. Rev. Lett., 72,3262 . 